\title{
Study on haemoglycemic effects of xylazine, diazepam and ketamine in surgically treated dogs
}

\author{
Wajid Ali Khan ${ }^{1}$, UzmaFarid Durrani ${ }^{2}$, Sadaf Aslam ${ }^{1}$, Aqeel Javeed ${ }^{3}$, \\ Asim Khalid Mahmood ${ }^{2}$ Muhammad Waqas ${ }^{2}$, \\ ${ }^{I}$ Department of Clinical Medicine and Surgery, Faculty of Veterinary Science, University of Veterinary and \\ Animal Sciences, Lahore, Pakistan \\ ${ }^{2}$ Pet Centre, University of Veterinary and Animal Sciences, Lahore, Pakistan \\ ${ }^{3}$ Department of Pharmacology and Toxicology, Faculty of Bio-Sciences, University of Veterinary and Animal
}

Sciences, Lahore, Pakistan

\begin{abstract}
Individual effects of xylazine, diazepam and ketamine were studied on haemoglycemic values of 30 minor surgical cases of adult dogs. Dogs having similar weight and age were divided into three groups $(A, B, C)$, each with 10 dogs. Groups $A, B$ and $C$ were treated with selected dosages of xylazine $(1.1 \mathrm{mg} / \mathrm{kg}, i . v)$, diazepam $(0.5 \mathrm{mg} / \mathrm{kg}$, i.v) and ketamine $(10 \mathrm{mg} / \mathrm{kg}$, i.v), respectively. Blood sampling was carried at regular time intervals before and after drug administration as follows: 0 minute, 30 minutes, 1 hour, 2 hours, 4 hours, 6 hours, and 8 hours followed by serological assay using 'Glucose Liquicolor kit' to determine the haemoglycemic values. Test results revealed that xylazine, diazepam and ketamine cause significant increase in serum glucose level as compared to the base line reference value when used intravenously $(P<0.05)$. It is concluded that xylazine, diazepam and ketamine along with the surgical stress raise the haemoglycemic level so extra care should be taken when using these drugs especially in cases already showing high haemoglycemic values.
\end{abstract}

Keywords:Diazepam, Glucose Liquicolor kit, Haemoglycemic effects, Ketamine, Xylazine,

\section{Introduction}

Clinical use of xylazine, an alpha-2-adrenergic agonist, has been a long standing practice throughout the word. Most striking reasons for the use of xylazine include its safe sedative efficacy, easy availability and economical price [5].Alpha-2-adrenergic agonists are also used in combination with ketamine at reduced doses as compared to their individual doses to induce a good quality general anesthesia and to avoid emergence of various side effects associated with individual use of xylazine and ketamine [22].It has been observed thatblood profilechanges which occur, during and after the surgical procedures, critically affect the surgical outcomes[1].One of these changes is raiseinhaemoglycemic level after anaesthesia administration. There are various factors that can disturb blood glucose level during the surgery and effect of sedatives is very important among these factors [2]. Adrenaline and nor adrenaline maintain normal glucose level in body which effects both production as well as disposal of glucose throughout the body [3]. Surgical stress is often accompanied by hormonal and metabolic changes (catabolism of stored fats), and the proportion of these changes depends on the extent of procedure and existing disorder [4]. Xylazine is commonly used in veterinary field for different purposes, most commonly as sedative, analgesic and muscle relaxant. Studies on the effect of alpha-2-adrenergic agonists have proved that these agents cause decrease in plasma catecholamine concentrations that resultantly inhibit insulin release and ultimately lead to increased plasma glucose level [6]. Ketamine increases the blood glucose level in cats undergoing surgery as compared to the cats having no surgical stress [7]. Anesthetic agents along with the surgical procedures can increase the blood glucose level and can disturb the surgical outcomes [8]. Diazepam belongs to the benzodiazepine group. It is a potent tranquilizer that is used alone as well as in combination with other agents. Haemoglycemic effects of diazepam are not fully known. Present study was designed to see the long term haemoglycemic effect of xylazine, diazepam and ketamine in surgically treated dogs.

\section{Materials And Methods}

This clinical study was conducted on 30 adult dogs of either sex undergoing different minor surgical procedures (wound closure, abscess drainage, hematoma correction, fracture reduction, cast application and dental extraction) at outdoor clinic of Pet Centre, University of Veterinary and Animal Sciences, Lahore, Pakistan. All dogs were divided into three groups titled A, B and C; each group with 10 dogs. Complete physical examination was carried out for each dog to determine the general health status prior to anaesthesia administration and surgical procedure. All dogs were kept off feed 12 hours prior to the drug administration. In 
group A, dogs were treated with xylazine $(1.1 \mathrm{mg} / \mathrm{kg}$ i.v), in group B, dogs were treated with diazepam $(0.5 \mathrm{mg} / \mathrm{kg}$, i.v) while dogs in group C were treated with ketamine $(10 \mathrm{mg} / \mathrm{kg}$, i.v $)$.

\subsection{Sample collection and handling}

Each dog was implanted with venous cannula in cephalic vein for blood sample collection. $1 \mathrm{~mL}$ blood sample was collected for each test. First sample was collected prior to the drug administration to determine baseline reference value of serum glucose level. After drug administration sampling was carried out at different time intervals during and after the surgical procedure. Sampling intervals were: 0 minute, 30 minutes, $1 \mathrm{hr}, 2$ hrs, $4 \mathrm{hrs}, 6 \mathrm{hrs}$, and $8 \mathrm{hrs}$ [2]. Each blood sample was immediately shifted to the gel clot activator vacutainer followed by serum separation. Serum was then shifted to the eppendorf tube using micropipette. Samples were then processed at Pet Centre Diagnostic Laboratory, University of Veterinary and Animal Sciences, Lahore, Pakistan, for analysis of serum glucose levels.

\subsection{Statistical analysis}

Data were statistically analyzed using one way analysis of variance (ANOVA) for repeated measures and confidence interval was selected as $95 \%$. P value $<0.05$ was considered significant.

\section{Results}

Mean haemoglycemic values of Group A at different intervals, are presented in TABLE 1. In group A, xylazine induced smooth sedation with good analgesia and muscle relaxation. Statistical analysis of haemoglycemic data at various intervals revealed a significant difference among haemoglycemic values till 2 hours $(\mathrm{P}<0.05)$ followed by no significant difference till 8 hours $(\mathrm{P}>0.05)$, TABLE 1 . In group B,diazepam induced a mild sedation of a short duration and moderate analgesia. Statistical analysis of various haemoglycemic values revealed a significant difference among haemoglycemic values till 1 hour $(\mathrm{P}<0.05)$ followed by no significant difference till $8 \mathrm{hrs}$. ( $\mathrm{P}>0.05)$, TABLE 2 . In group $\mathrm{C}$, ketamine induced moderate anaesthesia with deep analgesia but poor muscle relaxation. Statistical analysis of various haemoglycemic values in group $\mathrm{C}$ showed a significant difference among haemoglycemic values till 2 hour $(\mathrm{P}<0.05)$ and later on there was no significant difference till 8 hrs. ( $\mathrm{P}>0.05)$,TABLE 3 .

\section{Discussion}

This study was designed to evaluate the long term individual effects of xylazine and diazepam as sedatives and ketamine anesthetic in clinical cases of dogs undergoing minor surgical procedures. It has been observed that when haemoglycemic level is raised beyond the normal values it affects the immune system by disturbing the process of pathogens killing, phagocytosis, antibodies (immunoglobulin) glycosylation and decrease in neutrophils [9]. Because of increased protein catabolism prognosis of skin grafting is often affected by high haemoglycemic level [10]. In this under discussion study it was observed that the use of ketamine along with surgical stress elevates the haemoglycemic values[8]. Stress activates hypothalamus which in turn stimulates the release of adrenergic hormones [1] and activates the sympathetic nervous system part of autonomic nervous system. Release of adrenergic hormones activates adrenal glands (medullary portion) and epinephrine is released [11] which in turn activates the process of glycogenolysis in muscles as well as in liver [1]. Stress also activates the corticotropic releasing hormone (CRH) following the stimulation of hypothalamus. This hormone then stimulates ACTH and affects adrenal gland which then releases cortisol. Cortisol causesglycogenolysis in muscles as well as in liver [1]. Findings of the present study are in line with the earlier reported studies. Current study revealed the same hyperglycemic effects of intravenously administered ketamine. This increase in haemoglycemic level due to ketamine could be either due to the stress or suppression of insulin hormone or might be due to the elevated concentration of cortisol [12]. Ketamine has similar haemoglycemic effect as that of alpha-2-adrenoceptor agonist drugs i.e. xylazine, detomidine and medetomidine [12]. A recent study on ketamine showed that haemoglycemic effect of the dissociative anesthetic depends on the dosage of the agent [13].

It has been observed in various studies that alpha-2-adrenoreceptor agonist xylazine increases the haemoglycemic level in cattle [18] as well as in rats [15] depending upon its dosage. It has been reported on the basis of previous studies that when xylazine is administered at a dosage of $2.2 \mathrm{mg} / \mathrm{kg}$, intramuscularly, haemoglycemic level increases and insulin concentration decreases [14]. Findings of the present study have confirmed that xylazine alone elevates the haemoglycemic level when administered at a dosage of $1.1 \mathrm{mg} / \mathrm{Kg}$, intravenously $(\mathrm{P}<0.05)$ as compared to the normal baseline values. This increase was due to the reduced secretion of insulin hormone. [19] has documented the same findings in feline while [18] also documented the same results in ovine. Besides insulin there are also some other hormones like glucagon which may affect the haemoglycemic level. Alpha-2-adrenoreceptor agonist xylazine elevates the concentration of these hormones in blood [20]. It is also documented earlier that xylazine and ketamine cocktail elevates haemoglycemic 
levelssignificantly in canine, ovine as well as in deer $[16,17]$. Earlier studies showed that diazepam alone increases the haemoglycemic levels in animals. Same findings are also documented in this study as elevated haemoglycemic level with the use of diazepam as $0.5 \mathrm{mg} / \mathrm{kg}$ when administered intravenously. Increase in haemoglycemic values occurred as a result of stimulation of adrenoreceptors ultimately leading to reduced insulin secretion, stress hormones may also be one of the contributing factors[21].

\section{Conclusion}

On the basis of findings of the present study it is concluded that intravenous use of xylazine, diazepam sedatives and ketamine anestheticelicit effect on haemoglycemic levels to a variable extent. It is recommended that a regular and continuous haemoglycemicmonitoring must be carried out at regular intervals throughout the preand post anesthetic period to rule out any possibility of unwanted and hazardous effects of hyperglycemia.

\section{References}

[1]. S M Genuth. Adrenal gland. In: Berne RM, Levy MM, Koeppen BM, Stanton BA (eds) Physiology. 5th edition. Philadelphia : Saunders; 2004,323-327.

[2]. H Fattahian, H Moosavian, H Mohyeddin, H Akbarin and R Moridpour, Blood glucose concentration profile after single dose of dexamethasone during operation in dogs, Comparative Clinical Pathology, 21, 2012, 957-960.

[3]. E Savontaus, V Fagerholm, O Rahkonen and M Scheinin, Reduced blood glucose levels, increased insulin levels and improved glucose tolerance in alpha2A-adrenoceptor knockout mice,European Journal of Pharmacoogyl, 578 (2-3), 2008, 359-364.

[4]. J PDesborough, The stress response to trauma and surgery. British Journal of Anaesthesia, 85 (1), 2008, 109-117.

[5]. T Beths, J B Glen, J Reid, A M Monteiro and A M Nolan, Evaluation and optimisation of target controlled infusion system for administering propofol to dogs as part of total intravenous anaesthetic technique during dental surgery, Veterinary Record. 148 (7), 2001 , 198-203.

[6]. T D Ambrisko and Y Hikasa. Neurohormonal and metabolic effects of medetomidine compared with xylazine in beagle dogs, Canadian Journal of Veterinary Research, 66 (1), 2002, 42-49.

[7]. L E Glerum , C M Egger, S W Allen and M Haag., Analgesic effect of the transdermal fentanyl patch during and after feline ovariohysterectomy, Veterinary Surgery, 30 (4), 2001, 351-358.

[8]. A Kumar, V K Sobti and K I Singh, Evaluation of haloperidol--ketamine mixture (1:1) anaesthesia in dogs, Journal of Veterinary Medecine A Physiology Pathology Clinical Medecine, 48 (2), 2008, 65-73.

[9]. C T Black, P J Hennessy and R J Andrassy, Short-term hyperglycemia depresses immunity through nonenzymatic glycosylation of circulating immunoglobulin, Journal of Trauma, 30, 1990, 830-832.

[10]. D C Gore, D Chinkes, JHeggers, D N Herndon, S E Wolf and M Desai, Association of hyperglycemia with increased mortality after severe burn injury, Journal of Trauma, 51, 2001, 540-544.

[11]. S I Sharif and H A Abouazra, Effect of intravenous ketamine administration on blood glucose levels in conscious rabbits, American Journal of Pharmacology and Toxicology, 4(2), 2009, 38-45.

[12]. S A Bergman, Ketamine: review of its pharmacology and its use in pediatric anesthesia,Anesthesia Progress, 46, 1999, 10-20.

[13]. J K Saha, J Xia, J M Grondin, S K Engle and J A Jakubowski, Acute hyperglycemia induced by ketamine/xylazine anesthesia in rats: mechanisms and implications for preclinical models, Experimenta Biology Medecine (Maywood), 230 (10), $2005,777-784$.

[14]. G J Benson, T L Grubb, C Neff-Davis, W A Olson, J C Thurmon, D L Lindner, W J Tranquilli, O Vanio. Perioperative stress response in the dog: effect of pre-emptive administration of medetomidine. Veterinary Surgery 29 (1), 2000, 85-91.

[15]. M Gotoh, A Iguchi, N Sakamoto. Central versus peripheral effect of clonidine on hepatic venous plasma glucose concentrations in fasted rats. Diabetes. 37 (1), 1988, 44-49.

[16]. Z B Ismail, K Jawasreh, A A Majali. Effects of xylazine-ketamine-diazepam anesthesia on blood cell counts and plasma biochemical values in sheep and goats. Comparitive Clinical Pathology, 19, 2010, 571-574.

[17] M C Stewart, A W English. The reversal of xylazine/ketamine immobilisation of fallow deer with yohimbine. Australian Veterinary Journal. 67 (9), 1990, 315-317.

[18]. R P Brockman. Effect of xylazine on plasma glucose, glucagon and insulin concentrations in sheep. Research in Veterinary Science, 30 (3), 1981, 383-384.

[19]. W Feldberg, H W Symonds. Hyperglycemic effect of xylazine. Journal of Veterinary Pharmacology Therapy, 3, 1980, 197-202.

[20]. Willard MD, Tvedten H, Turnwald GH. Small animal clinical diagnosis by laboratory methods. Philadelphia: Saunders; 1989; 186187.

[21]. J Yamada, Y Sugimoto, T Noma. Involvement of adrenaline in diazepam-induced hyperglycemia in mice. Life Sciences, 66 (13), 2000, 1213-1221.

[22] T Sano, R Nishimura, M Mochizuki, N Sasaki. Effects of midazolam-butorphanol, acepromazine-butorphanol and medetomidine on an induction dose of propofol and their compatibility in dogs, the Journal of Veterinary Medical Sciences, 65 (10), 2003, : 11411143 . 
Study on haemoglycemic effects of xylazine, diazepam and ketamine in surgically treated dogs

Table 1: Mean haemoglycemic values of group A

\begin{tabular}{|c|c|}
\hline \multirow{2}{*}{ Time } & Mean haemoglycemic values(mg/d) \\
\cline { 2 - 2 } & Group A \\
\hline 0 minute & $82.60 \pm 7.09$ \\
\hline 30 minutes & $127.0 \pm 6.0^{*}$ \\
\hline 1 hour & $121.6 \pm 2.07^{*}$ \\
\hline 2 hours & $129.8 \pm 5.06^{*}$ \\
\hline 4 hours & $87.00 \pm 6.04$ \\
\hline 6 hours & $85.20 \pm 9.17$ \\
\hline 8 hours & $88.20 \pm 5.44$ \\
\hline
\end{tabular}

* $\mathrm{P}<0.05$, data varies significantly from baseline value.

Table 2: Mean haemoglycemic values of groupB

\begin{tabular}{|c|c|}
\hline \multirow{2}{*}{ Time } & Mean haemoglycemic values(mg/dl) \\
\cline { 2 - 3 } & Group B \\
\hline 0 minute & $80.60 \pm 4.72$ \\
\hline 30 minutes & $95.00 \pm 4.30^{*}$ \\
\hline 1 hour & $90.40 \pm 5.63^{*}$ \\
\hline 2 hours & $83.80 \pm 3.34$ \\
\hline 4 hours & $82.80 \pm 3.11$ \\
\hline 6 hours & $81.60 \pm 6.98$ \\
\hline 8 hours & $79.80 \pm 8.28$ \\
\hline
\end{tabular}

$* \mathrm{P}<0.05$, the data varies significantly from baseline value.

Table 3: Mean haemoglycemic values of group C

\begin{tabular}{|c|c|}
\hline \multirow{2}{*}{ Time } & Mean haemoglycemic values(mg/dl) \\
\hline & Group C \\
\hline 0 minute & $80.00 \pm 8.63$ \\
\hline 30 minutes & $100.00 \pm 4.06^{*}$ \\
\hline 1 hour & $97.80 \pm 3.11 *$ \\
\hline 2 hours & $90.80 \pm 8.22 *$ \\
\hline 4 hours & $85.00 \pm 8.09$ \\
\hline 6 hours & $81.00 \pm 5.38$ \\
\hline 8 hours & $83.40 \pm 5.59$ \\
\hline
\end{tabular}

* $\mathrm{P}<0.05$, the data varies significantly from baseline value. 